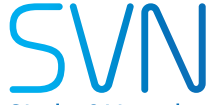

Stroke \& Vascular Neurology

\title{
A comparison between acute large vessel occlusion in the posterior circulation and anterior circulation after endovascular treatment: the ANGEL- ACT registry experience
}

Xuan Sun, Jingyu Zhang (i) , Xu Tong (1) , Baixue Jia (D), Dapeng Mo, Ning Ma, Feng Gao, Lian Liu, Xiaochuan Huo (D) , Ligang Song, Yiming Deng, Zhongrong Miao (i)

To cite: Sun X, Zhang J, Tong X, et al. A comparison between acute large vessel occlusion in the posterior circulation and anterior circulation after endovascular treatment: the ANGEL-ACT registry experience. Stroke \& Vascular Neurology 2022;0. doi:10.1136/svn-2021001093

- Additional supplemental material is published online only. To view, please visit the journal online (http://dx.doi.org/10. 1136/svn-2021-001093).

XS and JZ contributed equally.

XS and JZ are joint first authors.

Received 3 May 2021

Accepted 2 November 2021

Check for updates

(c) Author(s) (or their employer(s)) 2022. Re-use permitted under CC BY-NC. No commercial re-use. See rights and permissions. Published by BMJ.

Interventional Neuroradiology Center, Beijing Tiantan Hospital, Capital Medical University, Beijing, China

Correspondence to Professor Zhongrong Miao; miaozhongrong123@126.com

\section{ABSTRACT}

Background and purpose Endovascular treatment (EVT) has shown an overwhelming benefit for acute anterior circulation artery occlusion (ACO). Whether it can achieve the same outcomes in posterior circulation artery occlusion (PCO) has not been well explained. We aimed to evaluate the characteristics and prognosis of ACO and PCO after EVT in a nationwide registry.

Method The present analysis was based on the prospective ANGEL-ACT Registry in China between November 2017 and March 2019. Demographic data, periprocedural times, recanalisation rate, intracranial haemorrhage $(\mathrm{ICH})$ and 90-day functional outcomes were compared between the ACO and PCO groups.

Results A total of 1793 patients were analysed including 397 (22.1\%) consecutive patients with PCO and 1396 (77.9\%) patients with ACO treated with EVT. A larger proportion of patients with PCO had intracranial atherosclerotic disease and received extra angioplasty during EVT. Successful recanalisation and 90-day favourable functional outcomes did not differ significantly between the two groups. Patients with PCO showed lower 24-hour ICH and symptomatic ICH rates. There was a trend towards higher mortality rate in the PCO group $(22.09 \%$ vs $14.44 \%$; adjusted OR 1.286 (95\% Cl 0.820 to 2.017 ), $\mathrm{p}=0.2731$ ), especially when the onset to puncture time was over 6 hours $(30.77 \%$ vs $11.13 \%$; adjusted OR 2.673 (95\% Cl 1.280 to 5.583), $p=0.0089$, interactive $p=0.0002$ ). Conclusions In this large prospective multicentre registry, there was a significant difference in the characteristics and periprocedural features between patients with PCO and ACO. However, successful recanalisation and 90-day favourable functional outcomes in PCO were equivalent to those in ACO.

\section{INTRODUCTION}

Endovascular treatment (EVT) has shown an overwhelming benefit for acute anterior circulation artery occlusion (ACO).${ }^{1-7}$ Evidence on the efficacy and safety of EVT for posterior circulation artery occlusion (PCO) is still lacking. ${ }^{8}$ With PCO, however, acute ischaemic strokes (AIS) rarely occur with varied presentation and remain devastating with a high mortality and morbidity rate, especially without early reperfusion. ${ }^{9}$ With updates to EVT materials and the development of multimodal imaging evaluations, a larger number of clinical practices focus on EVT in PCO populations. ${ }^{10} 11$ Whether EVT can achieve the same outcomes in PCO as in ACO has not been well explained. We therefore analysed the data collected from a prospective multicentre registry ${ }^{12}$ to investigate differences in the characteristics of $\mathrm{ACO}$ and $\mathrm{PCO}$ and to ascertain the treatment prognosis between the two groups.

\section{METHOD}

\section{Patients' selection}

Written informed consent was obtained from all patients to participate in the study, and the privacy of the research subjects was strictly protected. The data of the patients included in this study were retrieved from the ANGEL-ACT Registry. The ANGEL-ACT Registry is a multicentric, nationwide, prospective registry study launched in December 2017 and terminated in March 2019. The characteristics and operating protocols of ANGEL-ACT Registry have already been published. ${ }^{12}$ The ANGEL-ACT Registry enrolled 2004 patients with AIS who had undergone EVT. Patients were enrolled according to the following inclusion criteria: (1) Age $\geq 18$ years; (2) Diagnosis of AIS caused by imaging-confirmed intracranial large vessel occlusion (LVO); and (3) Initiation of any type of EVT, including intra-arterial thrombolysis, mechanical thrombectomy (MT), angioplasty and permanent stenting. The exclusion criteria were (1) Isolated cervical internal carotid artery or vertebral 


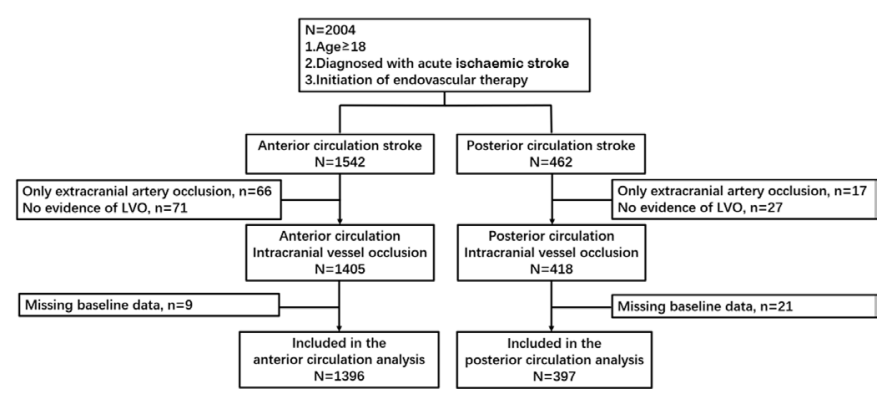

Figure 1 Flow chart of patient screening for the ANGELACT registry. LVO, large vessel occlusion.

artery (VA) occlusion and (2) No evidence of LVO angiogram. Among the enrolled patients, 83 patients with isolated extracranial artery occlusion, 98 patients with no evidence of LVO on digital subtraction angiography, and 30 patients with missing baseline data were excluded from the data analysis, leaving $397(22.1 \%)$ consecutive patients with PCO and 1396 (77.9\%) patients with ACO. A flow chart of patient screening is presented in figure 1.

\section{Imaging assessment}

All enrolled patients were required to complete brain plain CT scan before the procedure. The examination method of plain CT scan combined with CT angiography (CTA) was recommended for patients with ACO, and MRI and angiography (MRI/MRA) was recommended for patients with PCO. If priority examination cannot be performed due to the limitation of central conditions, the imaging evaluation of brain tissue and blood vessels can be completed according to the actual situation. The head plain CT scan must be completed $24 \pm 3$ hours after the procedure. If conditions permit, MRI/MRA can be performed to evaluate the final infarction volume, and CTA is preferred to evaluate whether there is vascular recanalisation or not.

\section{Data collection}

Data reporting preprocedural and procedural variables were extracted by reviewing the patient charts, procedure notes, angiograms, and progression and follow-up

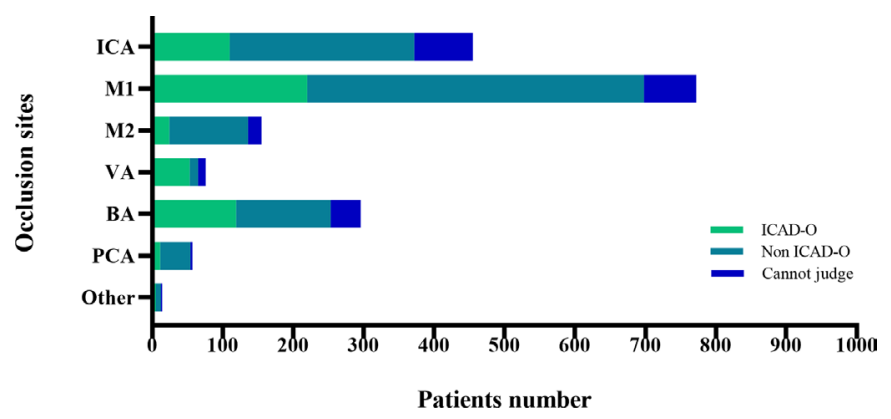

Figure 2 The distribution of artery occlusion site of the ANGEL-ACT registry. ICA, intracranial artery; ICAD$\mathrm{O}$,intracranial atherosclerotic disease related occlusion; $\mathrm{M} 1$, middle cerebral artery M1 segment; M2, middle cerebral artery M2 segment; BA, basilar artery; PCA, posterior cerebral artery; VA, vertebral artery. notes. Preprocedural variables included in this analysis were age, sex, administration of intravenous thrombolysis (bridging IVT), National Institute of Health Stroke Scale (NIHSS) Score on admission, preantiplatelet treatment, vascular risk factors (such as previous ischaemic stroke, hypertension, diabetes mellitus, atrial fibrillation, alcohol consumption, and previous or current smoking habit), and pathogenesis of stroke (large artery atherosclerosis, cardioembolism and others). Procedural variables included the time from symptom onset to puncture and recanalisation (OPT, ORT), the types of endovascular procedures (stent thrombectomy, intra-arterial thrombolysis, catheter aspiration, balloon angioplasty and permanent stenting) and administration of heparin $\mathrm{IIb} /$ IIIa receptor inhibitors during thrombectomy. Baseline and postprocedural modified Thrombolysis in Cerebral Infarction Scale Score, and occurrence of intracranial haemorrhage $(\mathrm{ICH})$ on post-treatment imaging were reviewed by core laboratory image interpretation. All patients' images were selected separately and reviewed by two interventionalists blinded to the patient details.

\section{Outcome measures}

The primary outcome was functional independence, defined as a modified Rankin Scale (mRS) Score of 0-2 at 90 days. The 90-day follow-up was ascertained using a standardised telephone interview performed by trained investigators blinded to the baseline and procedural data. Secondary outcomes included (1) Technical efficacy according to successful reperfusion rate, defined as a postprocedural modified Thrombolysis in Cerebral Infarction Scale Score of 2b or 3; (2) Any type of ICH at 24 hours; (3) Symptomatic ICH (sICH) according to the Heidelberg Bleeding Classification criteria at 24 hours; and (4) Mortality within 90 days.

\section{Statistical analysis}

All data were presented as the median (IQR) for continuous and ordinal variables and a number (percentage) for categorical variables. A comparison of the baseline and procedural characteristics between both groups was performed using the Mann-Whitney $\mathrm{U}$ test for continuous and ordinal variables and Pearson's $\chi 2$ test for categorical variables. A binary logistic regression model was used for multivariable analysis. Outcomes were further compared based on subgroups of OPT over or less than 6 hours. A multivariable model was constructed with variables with values of $\mathrm{p}<0.1$ in univariate analysis using forward inclusion. The $\mathrm{p}$ value for inclusion in the model was $<0.05$. All analyses were performed in SAS, V.9.4, software (SAS Institute, Cary, North Carolina, USA).

\section{RESULTS}

A total of 1793 patients were included in the analysis: $1396(77.9 \%)$ patients with ACO and $397(22.1 \%)$ patients with PCO. The distribution of occlusion sites in both $\mathrm{ACO}$ and $\mathrm{PCO}$ groups is shown in figure 2. Baseline characteristics are listed in table 1. Compared with 
Table 1 Baseline characteristics

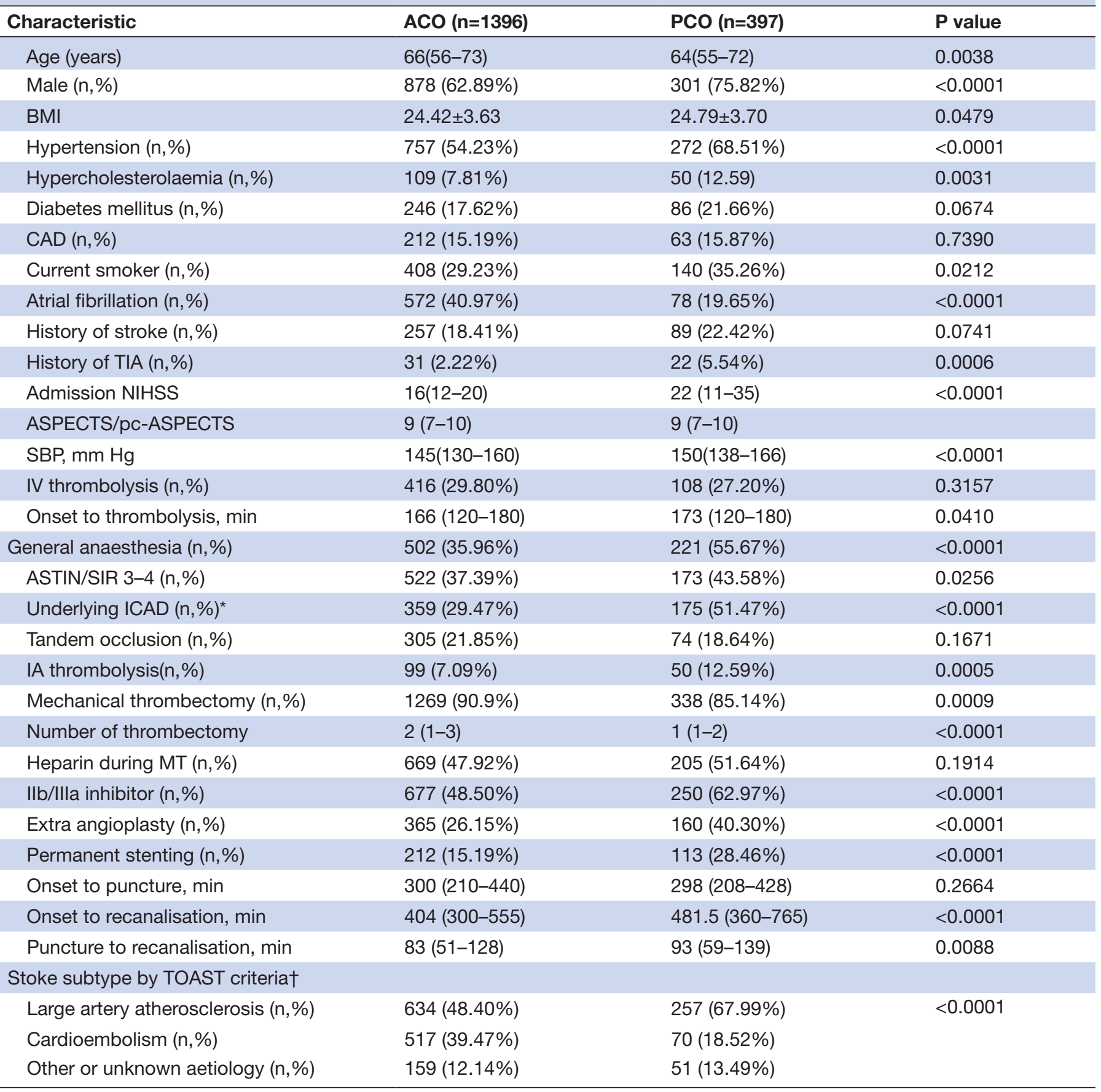

${ }^{*}$ Missing 235 data.

†Missing 105 data.

ACO, anterior circulation artery occlusion; ASPECTS, Alberta Stroke Programme Early CT Score; ASTIN/SIR, American Society of Interventional and Therapeutic Neuroradiology/Society of Interventional Radiology Collateral Flow Grading System; BMI, body mass index; CAD, coronary artery disease; IA, intra-artery; ICAD, intracranial atherosclerotic disease; IV, intravenous; MT, mechanical thrombectomy; NIHSS, National Institute of Health Stroke Scale; pc-ASPECTS, posterior circulation Acute Stroke Prognosis Early CT Score; PCO, posterior circulation artery occlusion; SBP, systolic blood pressure; TIA, transient ischaemic attack; TOAST, Trial of Org 10172 in Acute Stroke Treatment.

patients with ACO, those with PCO were more likely to be male $(75.8 \%$ vs $62.89 \%, \mathrm{p}<0.0001)$ and younger (64 years, IQR (55-72) vs 66 years, IQR (56-73), $\mathrm{p}=0.0038)$ and had a history of hypertension $(68.51 \%$ vs $54.23 \%, \mathrm{p}<0.0001)$, hypercholesterolaemia $(12.59 \%$ vs $7.81 \%, \mathrm{p}=0.0031)$, smoking $(35.26 \%$ vs $29.23 \%$, $\mathrm{p}=0.0212$ ) and transient ischaemic attack (TIA) (5.54\% vs $2.22 \%, \mathrm{p}=0.0006)$; higher NIHSS Score at admission (22, IQR (11-35) vs 16, IQR (12-20), $\mathrm{p}<0.0001)$; and longer ORT (481.5, IQR (360-765) vs 404 , IQR $(300-555), \mathrm{p}<0.0001)$. Furthermore, they were more likely to have intracranial atherosclerotic 


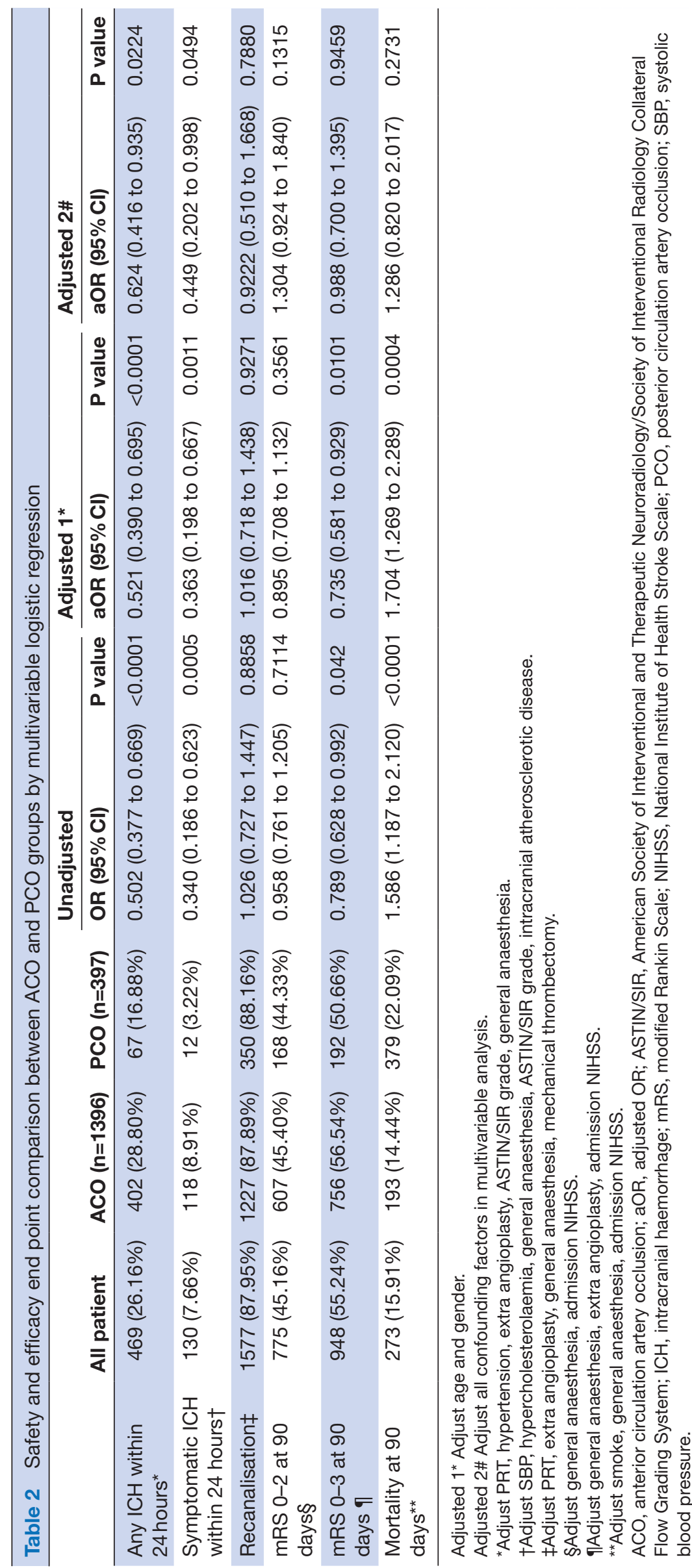




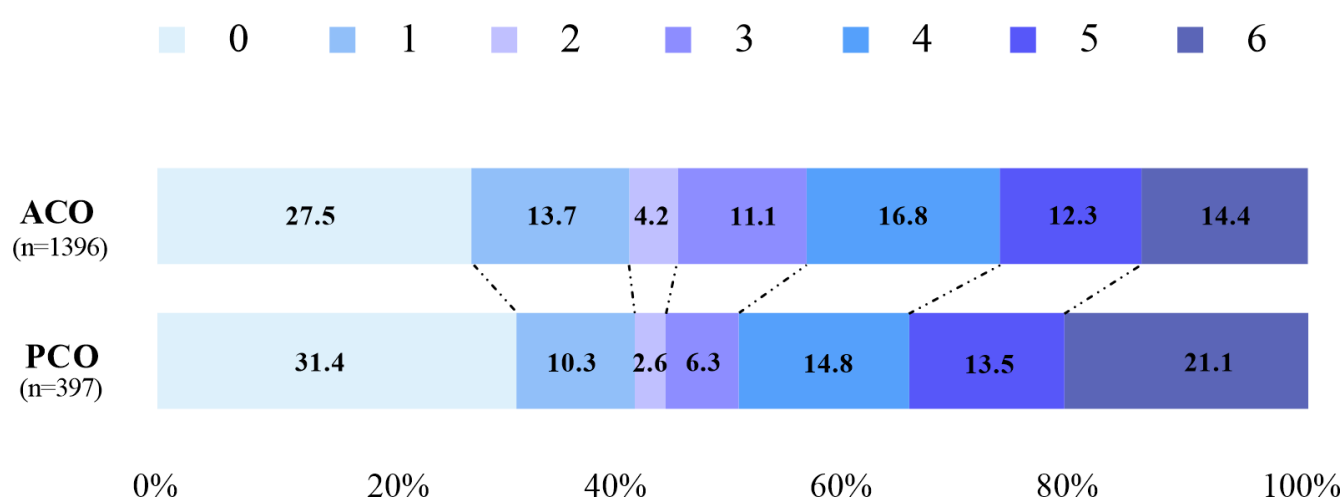

Figure 3 Outcome of the modified Rankin Scale at 90 days between the anterior circulation artery occlusion (ACO) and posterior circulation artery occlusion (PCO) groups.

disease (ICAD, $51.47 \%$ vs $29.47 \%, \mathrm{p}<0.0001)$ and receive intra-artery thrombolysis $(12.59 \%$ vs $7.09 \%$, $\mathrm{p}=0.0005)$ and IIb/IIIa receptor inhibitors $(62.97 \%$ vs $48.50 \%, \mathrm{p}<0.0001)$ and permanent stenting $(28.46 \%$ vs $15.19 \%, \mathrm{p}<0.0001)$ during EVT.

Regarding the pathogenesis of stroke, large artery atherosclerosis (67.99\%) was more often observed in the PCO group, while cardiac embolism $(18.52 \%)$ and other types of pathogeneses $(13.49 \%)$ were observed less often. Stent thrombectomy and catheter aspiration were performed more often in patients with ACO than in patients with PCO. Balloon angioplasty and permanent stenting were performed more often in patients with PCO than in those with ACO.

A comparison of recanalisation rates, postoperative haemorrhage and outcomes after a 90-day follow-up is shown in table 2. ICH, whether symptomatic or asymptomatic, occurred more frequently in ACO. PCO showed lower 24-hour ICH (16.88\% vs $28.80 \%$; OR $0.502(95 \%$ CI 0.377 to 0.669$), \mathrm{p}<0.0001$; adjusted OR 0.624 (95\% CI 0.416 to 0.935$), p=0.0224)$ and $\mathrm{sICH}(3.22 \%$ vs $8.91 \%$; OR 0.340 (95\% CI 0.186 to 0.623 ), $\mathrm{p}=0.0005$; adjusted OR 0.449 (95\% CI 0.202 to 0.998$), \mathrm{p}=0.0494$ ) rates than did ACO. Successful recanalisation and a favourable functional outcome at 90 days (mRS 0-2) did not differ significantly between $\mathrm{PCO}$ and ACO in either unadjusted or adjusted logistic regression $(88.16 \%$ vs $87.89 \%$ and $44.33 \%$ vs $45.40 \%$ ). The mRS distribution of the two groups is shown in figure 3 . It can be clearly seen from the data and graphs that there was a trend towards higher mortality rate in the $\mathrm{PCO}$ group than in the ACO group (22.09\% vs $14.44 \%$; OR 1.586 (95\% CI 1.187 to 2.120 ), $\mathrm{p}<0.0001$; adjusted OR 1.286 (95\% CI 0.820 to 2.017 ), $\mathrm{p}=0.2731$ ).

In the subgroup analysis, we found that patients with PCO showed similar independent functional outcomes (57.87\% vs $54.51 \%)$ and mortality $(15.32 \%$ vs $16.46 \%)$ at 90 days and a lower sICH risk within 24 hours than patients with ACO when OPT was less than 6 hours. However, when OPT was over 6 hours, PCO showed a devastating mortality rate compared with ACO $(30.77 \%$ vs $11.13 \%$; OR 3.548 (95\% CI 2.260 to 5.569), $\mathrm{p}<0.0001$; adjusted OR 2.673 (95\% CI 1.280 to 5.583), $\mathrm{p}=0.0089$, interactive $\mathrm{p}=0.0002$ ) (table 3 ).

Furthermore, multivariate logistic regression analysis adjusting for baseline characteristics was performed in the two groups separately. In the ACO group, age $<66$ years (OR 1.681 (95\% CI 1.255 to 2.252$)$ ), NIHSS Score $<16$ (OR 2.131 (95\%CI 1.594 to 2.850)), Alberta Stroke Programme Early CT Score (ASPECTS) $\geq 9$ (OR 1.489 (95\% CI 1.090 to 2.036)) and OPT $>6$ hours (OR 1.359 (95\% CI 1.006 to 1.837 )) were significant predictors for 90-day functional independence consistent with the majority of reported studies. While in the PCO group, we just found NIHSS Score <16 (OR 2.890 (95\% CI 1.773 to 4.694)) were a significant predictor for 90-day functional independence (figure 4). Finally, we compared the baseline characteristics and clinical outcomes of different occlusion sites, namely the VA-V4 segment, basilar artery (BA) and posterior cerebral artery (PCA) in the PCO group, and showed the results in the online supplemental table 1 and online supplemental table 2. Besides patients with PCA showed a lower successful recanalisation rate and no death at 90 days, other clinical outcomes did not relate to location site (online supplemental figure 1). A 90-day mRS distribution of VA, BA and PCA is shown in online supplemental figure 2 .

\section{DISCUSSION}

Our present study aimed to compare the clinical characteristics and outcomes between patients with PCO and those with ACO treated by EVT in real situations. In this report, we found that the pathogenesis of stroke and treatment procedures were significantly different in PCO and ACO, which affected their clinical outcome.

Similar to many other studies, ${ }^{13}{ }^{14}$ we found that large artery atherosclerosis and ICAD were more common in PCO than in ACO and that the larger percentage of men, higher BMI, and history of hypertension, hypercholesterolaemia, TIA and smoking related to ICAD were also observed in patients with PCO. Elderly patients and atrial fibrillation were more often observed in the ACO group. ${ }^{15}$ 


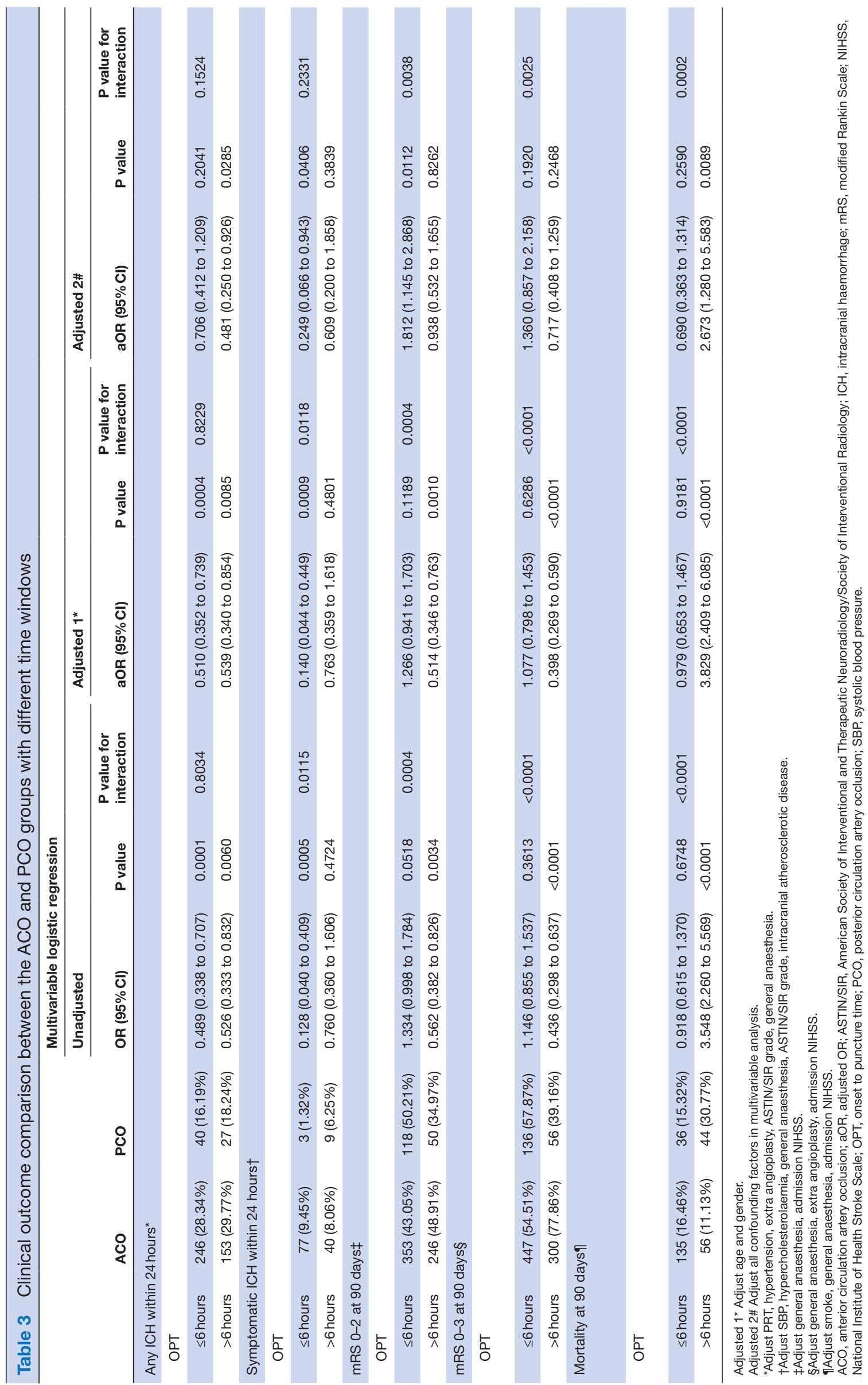



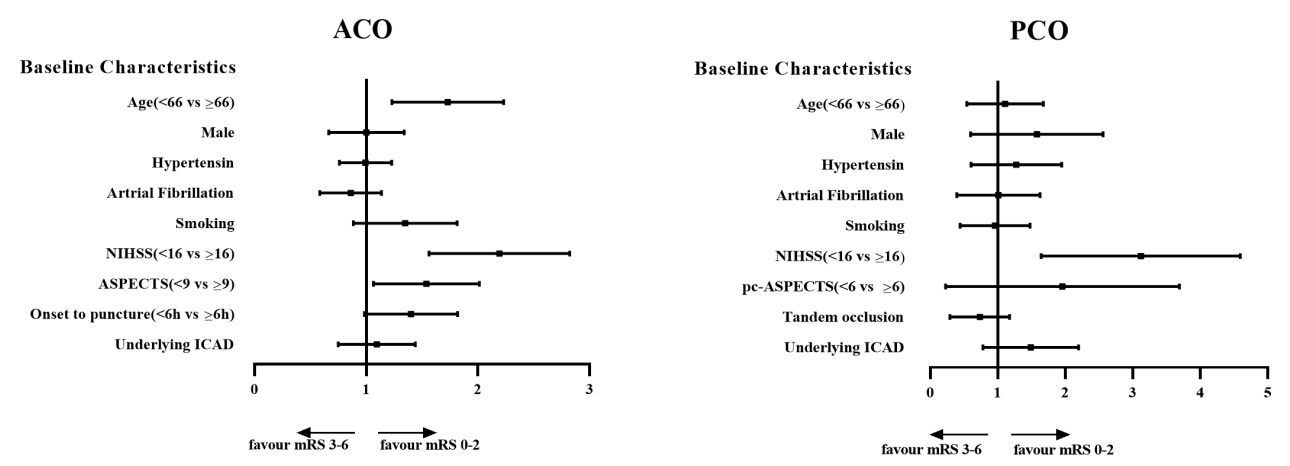

Figure 4 Predictors of a 90-day mRS Score 0-2 between the acute anterior circulation artery occlusion (ACO) and posterior circulation artery occlusion (PCO) groups. ASPECTS, Alberta Stroke Programme Early CT Score; ICAD, intracranial atherosclerotic disease; mRS, modified Rankin Scale; NIHSS, National Institute of Health Stroke Scale; pc-ASPECTS, posterior circulation Acute Stroke Prognosis Early CT Score.

Due to the significant difference in pathogenesis between the two groups, EVT strategies were not the same. MT is more frequently used to treat ACO, while the combination of intra-artery thrombolysis and/or IIb/ IIIa receptor inhibitors is more prevalent in PCO for resolving ICAD lesions. In our study, rescue angioplasty was also an important means for maintaining recanalisation, as balloon angioplasty and permanent stents are more commonly used in patients with PCO. It is clear that the technique of successful recanalisation was satisfactory in both patients with $\mathrm{PCO}$ and patients with ACO, with success rates of $88.16 \%$ and $87.89 \%$, respectively, slightly higher than those reported by a meta-analysis. ${ }^{16}$ The independent functional outcomes at 90 days seemed to be equivalent between the PCO and ACO groups $(44.33 \%$ vs $45.40 \%$ ). We were glad to see that the efficacy of properly performed EVT in patients with PCO could achieve similar outcomes to that in patients with ACO in real clinical practice.

Even with comparable successful recanalisation rates and independent functional outcomes, patients with PCO thrombectomy had relatively worse outcomes than those with ACO, as shown by a higher rate of mortality in our analysis $(22.09 \%$ vs $14.44 \%)$. There was a high chance that futile recanalisation occurred in patients with PCO. Previous studies ${ }^{17-19}$ pointed out that age, stroke severity, baseline NIHSS Score and duration of the procedure were related to poor outcome in patients with PCO. Early symptoms of PCO are complex and not easy to recognise, and the time from onset to initiation of treatment is longer; these factors often result in ineligibility for intravenous thrombolysis and a delay of initiation of the EVT procedure..$^{20}$ Another reason for the high mortality rate of PCO might be attributable to disturbing brainstem symptoms, such as a comatose state, dysphagia, tracheostomy, hypostatic pneumonia and complications due to a long-term bedridden state. However, the PCO mortality in our study was slightly lower than that in a meta-analysis ${ }^{16}$ including 474 patients with PCO (26.6\%) and the EVT arm group in the Basilar Artery Occlusion Endovascular Intervention versus Standard Medical Treatment (BEST)
Trial $^{10}$ and The EVT for Acute Basilar Artery Occlusion Study (BASILAR) ${ }^{11}$ (33\% and $\left.47.3 \%\right)$.

ICAD may be the main determining factor that results in different outcomes between patients with PCO and patients with ACO. ICAD was more frequently observed in patients with $\mathrm{PCO}$ and perhaps played an absolutely different role in the mechanisms and outcomes of stroke in $\mathrm{PCO}$ and $\mathrm{ACO} .{ }^{20}$ A study compared the EVT procedural and clinical outcomes among patients with ACO with and without ICAD. The results showed that the recanalisation rate and clinical outcomes did not differ between patients with or without ICAD in ACO. ${ }^{21}$ Another study compared the recanalisation rate and clinical outcomes of MT in different subtypes of basilar artery occlusion (BAO), and they found that $\mathrm{BAO}$ with in situ atherosclerotic thrombosis had a worse recanalisation rate and poorer clinical outcome than embolic BAO. ${ }^{22}$ In our study, we also found that more patients suffered TIAs before stroke in PCO. With stroke recurrence, the risk of cumulative disability and poor prognosis increases accordingly, ${ }^{23}$ which may be a reason for a poor outcome in PCO. Therefore, we should strengthen the identification and intervention of patients with ICAD in the early stage, especially for those who present with symptomatic posterior circulation ICAD and suffer recurrent TIAs. Early angioplasty may be reasonable and should be considered from a more positive perspective.

Similar to other research findings, ${ }^{24}$ patients with PCO also showed a lower rate of ICH than patients with ACO, including sICH and any type of ICH, even though intraartery thrombolysis and IIb/IIIa receptor inhibitors were more commonly used in our study. This might be explained by the larger volume of ischaemic tissue in ACO, which usually results from cardiac embolism, and oral preanticoagulant agents, which are also a risk factor. ${ }^{25}$ Compared with ACO, PCO typically causes smaller ischaemic lesion volumes, which may reduce the rate of ICH after EVT. In our study, we found that there are more patients with good collateral circulation presented American Society of Interventional and Therapeutic Neuroradiology/Society of Interventional Radiology Score 3-4 grade in PCO than 


\section{ACO}

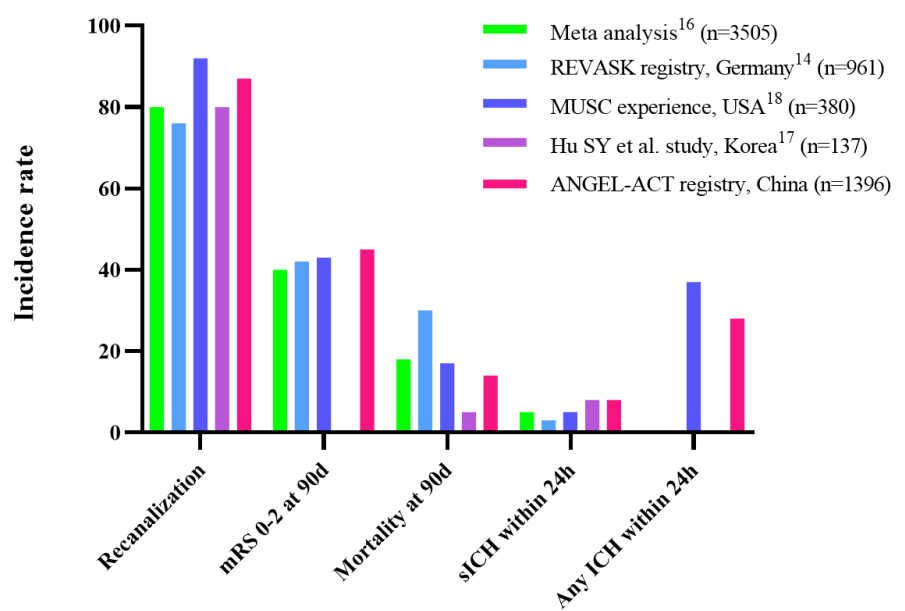

PCO

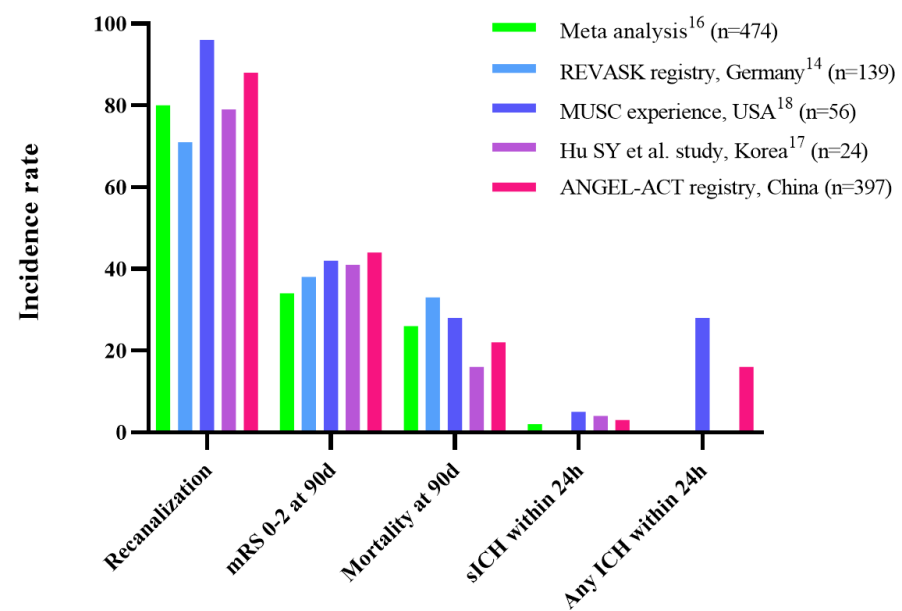

Figure 5 The clinical outcomes compared with other published studies. ACO, anterior circulation artery occlusion; ICH, intracranial haemorrhage; mRS, modified Rankin Scale; PCO, posterior circulation artery occlusion; sICH, symptomatic intracranial haemorrhage.

ACO (43.58\% vs $37.39 \%$, $\mathrm{p}=0.0256$ ), which maybe result in a lower chance of ischaemia-reperfusion injury after recanalisation. A study reported that blood-brain barrier derangements were more frequently observed in ACO $(22 \%)$ than in PCO (7\%). ${ }^{26}$ Another reason is that the number of MT device passes is higher in patients with ACO, which has been verified to be associated with ICH after EVT. ${ }^{27}$ We present a brief comparison of our findings with other studies ${ }^{14-18}$ in figure 5 .

Subgroup analysis according to the OPT time window gave rise to different functional outcomes. We found that successful recanalisation, 90-day favourable functional outcome and mortality in PCO were equivalent to ACO within a 6-hour OPT time window. However, when OPT was over 6 hours, the risk of 90-day death in PCO was 2.673 times higher than that in ACO. The test for interaction showed a significant difference in the clinical outcomes affected by ischaemic location at different OPT times.

Regardless of anterior and posterior circulation infarction, EVT in the early stage of onset is more to save the brain tissue in the ischaemic penumbra, while the collateral circulation in the PCO is better, which can quickly restore brain function and achieve the same clinical prognosis as the ACO. However, as the time of cerebral ischaemia is prolonged, the brainstem infarction caused by the progression of PCO often causes malignant cerebral infarction, brain herniation, death and other events that badly affect the clinical prognosis of patients, resulting in totally different clinical outcomes compared with ACO. This means that a selected OPT time window should be applied for assessment at admission for both patients with ACO and patients with PCO.

Finally, we tried to assess the potential predictive factors related to the 90-day independent outcome of ACO and PCO groups separately by multivariable analysis. The relevant data of the ACO group have been presented in our formerly published paper. And we found age $<66$ years,
NIHSS Score $<16$, ASPECTS $\geq 9$ were significant predictors for 90-day functional independence consistent with the majority of reported studies. However, in the PCO group, we just found patients with lower NIHSS Score have a towards to a better clinical outcome. It is possible that in the PCO group, disease severity and brainstem infarction play a greater role in prognosis. Since we only adjusted baseline data in the multivariable analysis, there perhaps exist other possible predictors that need to be figured out in our later research.

Some limitations should also be mentioned before generalising the present findings. First, this was not a randomised study, and measured or unmeasured variables may represent potential confounders that cannot be ruled out despite adjustment. Second, this study was exclusively conducted in the Chinese population. Delays in the process of EVT in China and the variety and complexity of surgical procedures, as well as the high proportion of ICAD in Chinese patients, more rescue balloon angioplasty and permanent stenting, and differences in perioperative antithrombotic therapy will affect the extrapolation of the results of our study to other populations. The sample size of patients with PCO was relatively small compared with that of patients with ACO. We compared the different characteristics and prognoses between ACO and PCO, which could be analysed separately in later trials.

\section{CONCLUSION}

Although the pathogenesis, anatomy and EVT strategies are different, with timely recanalisation, patients with PCO could obtain a similar clinical benefit to that of patients with ACO.

Acknowledgements The authors thank all relevant clinicians, statisticians and imaging technicians. 
Contributors ZM designed and led the study, responsible for the overall content as the guarantor. XS and JZ analyzed the data and prepared the first draft of the report. XS and JZ contributed equally to the work. XT and BJ were responsible for acquisition of data. DM, NM, FG, LL, XH, LS and YD were involved in revising the article for important intellectual content. All authors critically reviewed the article and approved the final version.

Funding This study was supported by the National Key Research and Development Program of China (Grant number 2016YFC1301501).

Competing interests The authors declare that the research was conducted in the absence of any commercial or financial relationships that could be construed as a potential conflict of interest.

Patient consent for publication Not applicable.

Ethics approval The protocol was approved by the Institutional Review Board of Beijing Tiantan Hospital, Capital Medical University (approval ID: KY-2017-048-01). Provenance and peer review Not commissioned; externally peer reviewed. Data availability statement Data are available upon reasonable request. The data that support the findings of this study are available on request from the corresponding author. The data are not publicly available due to privacy or ethical restrictions.

Open access This is an open access article distributed in accordance with the Creative Commons Attribution Non Commercial (CC BY-NC 4.0) license, which permits others to distribute, remix, adapt, build upon this work non-commercially, and license their derivative works on different terms, provided the original work is properly cited, appropriate credit is given, any changes made indicated, and the use is non-commercial. See: http://creativecommons.org/licenses/by-nc/4.0/.

\section{ORCID iDs}

Jingyu Zhang http://orcid.org/0000-0001-5723-9272

Xu Tong http://orcid.org/0000-0002-3277-6941

Baixue Jia http://orcid.org/0000-0001-8782-5344

Xiaochuan Huo http://orcid.org/0000-0003-1264-5132

Zhongrong Miao http://orcid.org/0000-0002-0165-9814

\section{REFERENCES}

1 Berkhemer OA, Fransen PSS, Beumer D, et al. A randomized trial of intraarterial treatment for acute ischemic stroke. $N$ Engl J Med 2015;372:11-20.

2 Campbell BCV, Mitchell PJ, Kleinig TJ, et al. Endovascular therapy for ischemic stroke with perfusion-imaging selection. $N$ Engl J Med 2015;372:1009-18.

3 Goyal M, Demchuk AM, Menon BK, et al. Randomized assessment of rapid endovascular treatment of ischemic stroke. $N$ Engl J Med 2015;372:1019-30.

4 Jovin TG, Chamorro A, Cobo E, et al. Thrombectomy within 8 hours after symptom onset in ischemic stroke. $N$ Engl J Med 2015;372:2296-306.

5 Saver JL, Goyal M, Bonafe A, et al. Stent-retriever thrombectomy after intravenous t-PA vs. t-PA alone in stroke. $N$ Engl J Med 2015;372:2285-95.

6 Albers GW, Marks MP, Kemp S, et al. Thrombectomy for stroke at 6 to 16 hours with selection by perfusion imaging. N Engl J Med 2018;378:708-18.

7 Nogueira RG, Jadhav AP, Haussen DC, et al. Thrombectomy 6 to 24 hours after stroke with a mismatch between deficit and infarct. $N$ Engl J Med 2018;378:11-21.

8 Schonewille WJ, Wijman CAC, Michel P, et al. Treatment and outcomes of acute basilar artery occlusion in the basilar artery international cooperation study (basics): a prospective registry study. Lancet Neurol 2009;8:724-30.

9 Schonewille WJ, Algra A, Serena J, et al. Outcome in patients with basilar artery occlusion treated conventionally. J Neurol Neurosurg Psychiatry 2005;76:1238-41.

10 Liu X, Dai Q, Ye R, et al. Endovascular treatment versus standard medical treatment for vertebrobasilar artery occlusion (best): an open-label, randomised controlled trial. Lancet Neurol 2020;19:115-22.

11 Writing group for the BG, Zi W, Qiu Z, et al. assessment of endovascular treatment for acute basilar artery occlusion via a nationwide prospective registry. JAMA Neurol 2020;77:561-73.

12 Jia B, Ren Z, Mokin M, et al. Current status of endovascular treatment for acute large vessel occlusion in China: a real-world nationwide registry. Stroke 2021;52:STROKEAHA120031869.

13 Meinel TR, Kaesmacher J, Chaloulos-lakovidis P, et al. Mechanical thrombectomy for basilar artery occlusion: efficacy, outcomes, and futile recanalization in comparison with the anterior circulation. $J$ Neurointerv Surg 2019;11:1174-80.

14 Weber R, Minnerup J, Nordmeyer H, et al. Thrombectomy in posterior circulation stroke: differences in procedures and outcome compared to anterior circulation stroke in the prospective multicentre REVASK registry. Eur J Neurol 2019;26:299-305.

15 Huo X, Gao F, et al, Raynald. Characteristic and prognosis of acute large vessel occlusion in anterior and posterior circulation after endovascular treatment: the angel registry real world experience. $J$ Thromb Thrombolysis 2020;49:527-32.

16 Wang F, Wang J, He Q, et al. Mechanical Thrombectomy for Posterior Circulation Occlusion: A Comparison of Outcomes with the Anterior Circulation Occlusion - A Meta-Analysis. J Atheroscler Thromb 2020;27:1325-39.

17 Hu SY, Yi HJ, Lee DH, et al. Effectiveness and safety of mechanical thrombectomy with stent Retrievers in basilar artery occlusion: comparison with anterior circulation occlusions. J Korean Neurosurg Soc 2017;60:635-43.

18 Alawieh A, Vargas J, Turner RD, et al. Equivalent favorable outcomes possible after thrombectomy for posterior circulation large vessel occlusion compared with the anterior circulation: the MUSC experience. J Neurointerv Surg 2018;10:735-40.

19 Uno J, Kameda K, Otsuji R, et al. Mechanical thrombectomy for basilar artery occlusion compared with anterior circulation stroke. World Neurosurg 2020;134:e469-75.

20 Ausman JI, Liebeskind DS, Gonzalez N, et al. A review of the diagnosis and management of vertebral basilar (posterior) circulation disease. Surg Neurol Int 2018;9:106.

21 Baek J-H, Kim BM, Heo JH, et al. Outcomes of endovascular treatment for acute intracranial Atherosclerosis-Related large vessel occlusion. Stroke 2018;49:2699-705.

22 Baik SH, Park HJ, Kim J-H, et al. Mechanical thrombectomy in subtypes of basilar artery occlusion: relationship to recanalization rate and clinical outcome. Radiology 2019;291:730-7.

23 Wang Y, Zhao X, Liu L, et al. Prevalence and outcomes of symptomatic intracranial large artery stenoses and occlusions in China: the Chinese intracranial atherosclerosis (CICAS) study. Stroke 2014;45:663-9.

24 Tong X, Liao X, Pan Y, et al. Intravenous thrombolysis is more safe and effective for posterior circulation stroke: data from the thrombolysis implementation and monitor of acute ischemic stroke in China (TIMS-China). Medicine 2016;95:e3848.

25 Spence JD. Cardioembolic stroke: everything has changed. Stroke Vasc Neurol 2018;3:76-83.

26 Lee M, Saver JL, Alger JR, et al. Blood-Brain barrier permeability derangements in posterior circulation ischemic stroke: frequency and relation to hemorrhagic transformation. J Neurol Sci 2012;313:142-6.

27 Hao $\mathrm{Y}$, Yang D, Wang $\mathrm{H}$, et al. Predictors for symptomatic intracranial hemorrhage after endovascular treatment of acute ischemic stroke. Stroke 2017;48:1203-9. 